\title{
ARTICLE \\ Niche rather than origin dysregulates mucosal Langerhans cells development in aged mice
}

Yael Horev ${ }^{1,2}$, Rana Salameh ${ }^{1}$, Maria Nassar ${ }^{1}$, Tal Capucha ${ }^{1}$, Yasmin Saba ${ }^{1}$, Or Barel ${ }^{1}$, Khaled Zubeidat ${ }^{1}$, Daniela Matanes ${ }^{1}$, Amit Leibovich ${ }^{1}$, Oded Heyman ${ }^{2}$, Luba Eli-Berchoer ${ }^{1}$, Salem Hanhan ${ }^{1}$, Gili Betser-Cohen ${ }^{3}$, Hagit Shapiro ${ }^{4}$, Eran Elinav ${ }^{4}$, Herve Bercovier ${ }^{5}$, Asaf Wilensky ${ }^{2}$ and Avi-Hai Hovav ${ }^{1}$

Unlike epidermal Langerhans cells (LCs) that originate from embryonic precursors and are self-renewed locally, mucosal LCs arise and are replaced by circulating bone marrow (BM) precursors throughout life. While the unique lifecycle of epidermal LCs is associated with an age-dependent decrease in their numbers, whether and how aging has an impact on mucosal LCs remains unclear. Focusing on gingival LCs we found that mucosal LCs are reduced with age but exhibit altered morphology with that observed in aged epidermal LCs. The reduction of gingival but not epidermal LCs in aged mice was microbiota-dependent; nevertheless, the impact of the microbiota on gingival LCs was indirect. We next compared the ability of young and aged BM precursors to differentiate to mucosal LCs. Mixed BM chimeras, as well as differentiation cultures, demonstrated that aged BM has intact if not superior capacity to differentiate into LCs than young BM. This was in line with the higher percentages of mucosal LC precursors, pre-DCs, and monocytes, detected in aged BM. These findings suggest that while aging is associated with reduced LC numbers, the niche rather than the origin controls this process in mucosal barriers.

Mucosal Immunology (2020) 13:767-776; https://doi.org/10.1038/s41385-020-0301-y

\section{INTRODUCTION}

Langerhans cells (LCs) are unique antigen presenting cells residing in external and mucosal stratified epithelia such as the epidermis and the oral epithelium. ${ }^{1}$ Besides their similar anatomical location, epidermal and mucosal LCs share transcriptomic signatures, phenotype, and certain immunological functions, ${ }^{2-4}$ but differ in their ontogeny. Whereas epidermal LCs originate from embryonic precursors (i.e. yolk sac macrophages and fetal liver monocytes), mucosal LCs arise from adult bone marrow precursors (i.e. pre-DCs and monocytes). ${ }^{5,6}$ Under inflammatory conditions, epidermal LCs can also differentiate from adult monocytes ${ }^{7,8}$ that can give rise to long-term LCs. ${ }^{9}$ Moreover, murine epidermal LC precursors enter the developing epidermis during embryogenesis, proliferate rapidly after birth and differentiate to a radioresistant LC population that maintains itself locally via self-renewal. ${ }^{6}$ The precursors of mucosal LCs, on the other hand, enter the epithelium only after birth and differentiate locally to radiosensitive LCs that are continually replaced by circulating precursors. $^{3}$ Such dissimilar differentiation mechanisms are considered to reflect the distinct physiology of each epithelium, since in contrast to mucosal epithelia, the epidermis is sealed prenatally and it is not accessible to circulating precursors at steady state. Despite the abovementioned developmental dissimilarities, differentiation, and maintenance of both epidermal and mucosal LCs are mediated by TGF- $\beta 1$, BMP7 and their receptors
ALK5 and ALK3.,10-14 Yet, BMP7/ALK3 signaling was proposed to regulate the development of epidermal LCs in human, while its role in murine epidermal LCs is not completely understood. In addition, the microbiota was shown to shape the postnatal differentiation of mucosal but not epidermal LCs. ${ }^{2}$ This indicates that each type of epithelium adapts distinct mechanisms to recruit, differentiate, and maintain LCs in a manner that fits its physiological functions. ${ }^{4}$

Aging is associated with altered immune functions of stratified epithelia that can lead to increased vulnerability to infections and higher risk to develop squamous cell carcinoma. ${ }^{15-18}$ As the main sentinels of stratified epithelia, LCs play a major role in immune surveillance, and consequently deterioration of the LC network with age is likely to contribute to immune evasion and cancer. ${ }^{19-22}$ Previous studies have shown that the frequencies of murine epidermal LCs are reduced in aged mice, although their proliferation and apoptosis kinetics are comparable to young LCs. ${ }^{23,24}$ Aged and young epidermal LCs were also shown to have similar migratory capability, but phagocytosis as well as their ability to stimulate T cells are impaired with age. ${ }^{23,24}$ Reduced LC density was also reported in aged human skin, and their migratory capacity in vitro is decreased compared with young control. ${ }^{25,26}$ With regards to mucosal LCs, conflicting data reported that oral LCs in humans are either reduced or unchanged during aging. ${ }^{27-30}$ Furthermore, whereas works describing reduced oral LCs with age

\footnotetext{
${ }^{1}$ The Institute of Dental Sciences, Faculty of Dental Medicine, Hebrew University, Jerusalem, Israel; ${ }^{2}$ Department of Periodontology, Faculty of Dental Medicine, Hebrew University-Hadassah Medical Center, Jerusalem, Israel; ${ }^{3}$ The Lautenberg Center for Immunology and Cancer Research, Faculty of Medicine, Hebrew University, Jerusalem, Israel;

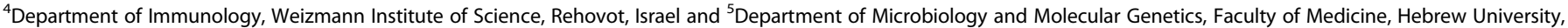
Jerusalem, Israel

Correspondence: Asaf Wilensky (asafw@ekmd.huji.ac.il) or Avi-Hai Hovav (avihaih@ekmd.huji.ac.il)

These authors contributed equally: Yael Horev, Rana Salameh

These authors jointly supervised this work: Asaf Wilensky, Avi-Hai Hovav
}

Received: 5 January 2020 Revised: 2 April 2020 Accepted: 19 April 2020

Published online: 26 May 2020 
a
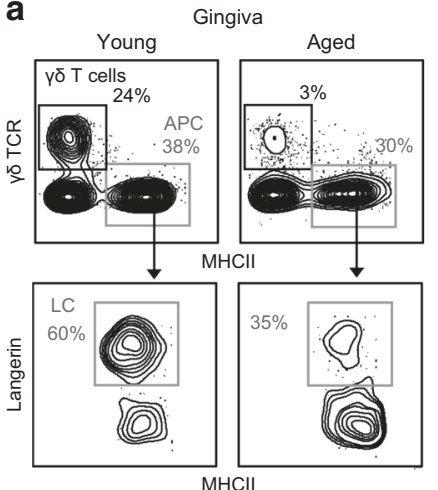
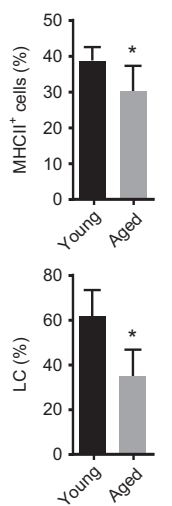
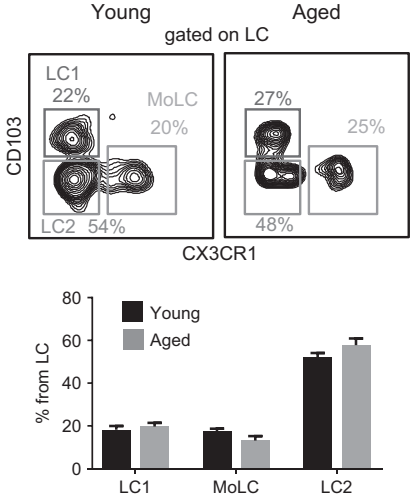

d

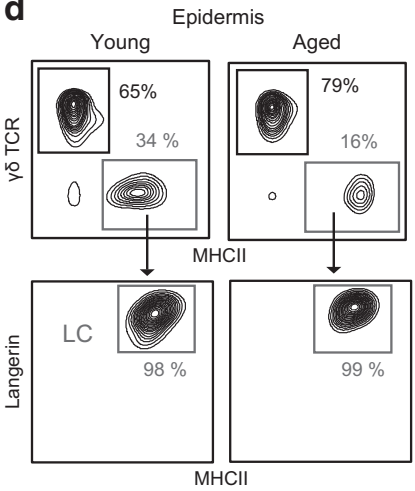

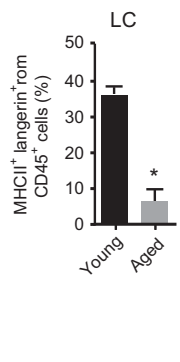

b

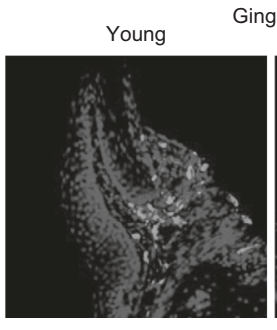
Gingiva
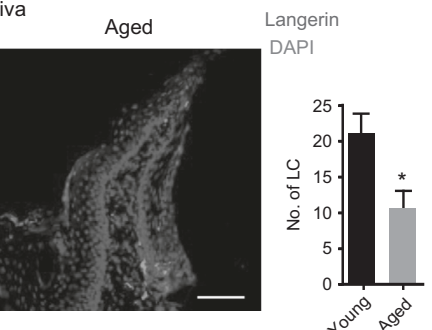

e
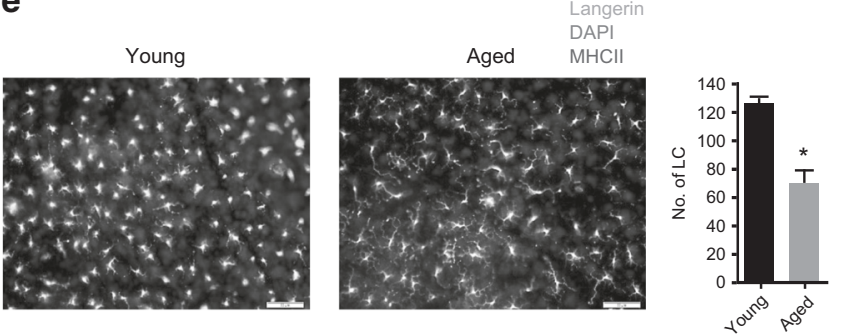

Fig. 1 Gingival LCs are reduced in aged mice. a Representative FACS plots demonstrate the identification of LCs in gingival epithelial tissues $\left(\mathrm{CD}_{4} 5^{+} \gamma \delta \mathrm{TCR}^{-} \mathrm{MHCII}^{+}\right.$langerin $\left.{ }^{+}\right)$of young and aged B6 mice, bar graphs show frequencies of $\mathrm{MHCII}^{+}$cells and LCs in these mice. Data are representative of four independent experiments with 5 mice/group. $\mathbf{b}$ Immunofluorescence staining of maxilla cross sections of young and aged B6 mice with mAb directed against langerin (red) and with DAPI (blue) for nuclear visualization. Bar graph shows the numbers of langerin-positive cells per field of view and represents the mean values of five mice (10 fields/mouse) + SEM. Representative data of three independent experiments. Scale bar $50 \mu \mathrm{m}$. c Representative FACS plots and graphs demonstrated the frequencies of the various LC subsets based on the expression of $\mathrm{CD} 103$ and $\mathrm{CX}_{3} \mathrm{CR} 1$, the cells were pre-gated on $\mathrm{LCs}\left(\mathrm{CD} 45^{+} \mathrm{MHCll}^{+} \mathrm{CD} 11 \mathrm{c}^{+} \mathrm{EpCAM}^{+}\right.$langerin $\left.{ }^{+}\right)$. Data are representative of three independent experiments with 3-5 mice/group (mean + SEM). d Representative FACS plots and graph depict the frequencies of $\mathrm{LCS}\left(\mathrm{CD}_{4} 5^{+} \gamma \delta \mathrm{TCR}^{-} \mathrm{MHCI}^{+}\right.$langerin $\left.{ }^{+}\right)$in the epidermis of young and aged mice. Data are representative of three independent experiments with five mice/group (mean + SEM). e Immunofluorescence staining of epidermal sheets of young and aged mice with mAb directed against langerin (green), MHCII (red) and with DAPI (blue). Bar graphs show the numbers of langerin-positive cells per field of view and represent the mean values of six mice (4 fields/mouse) + SEM. Representative data of three independent experiments. Scale bar $50 \mu m$. ${ }^{*} P<0.05$ (unpaired Student's $t$ test) compared with young samples.

also reported altered LC morphology in the gingiva and tongue, such alteration was not observed in other oral sites such as the buccal, lips, lateral border of the tongue and the hard palate. ${ }^{27}$ Contradictory data were also reported in mice by other studies that found either no change or reduced densities of oral LCs in aged mice. ${ }^{31,32}$ Taken together, these observations indicate that aging greatly affects epidermal LCs, whereas the impact on mucosal LCs is not yet resolved. To address the question whether and how aging regulates mucosal $\mathrm{LCs}$, we studied the differentiation of oral mucosal LCs during aging, focusing on both the niche and precursor levels. We propose that the niche, particularly via the microbiota, rather than the progenitors is dysregulating the development of LCs in aged mucosal epithelia.

\section{RESULTS}

Gingival LCs are reduced in aged mice but undergo distinct morphological changes compared with epidermal LCs To examine how aging affects mucosal LCs, we analyzed the presence of these cells in the gingival epithelium of 18- to 24month-old (aged) mice. Using flow cytometry (gating strategies are provided in Fig. S1A), we found that the frequencies and numbers of gingival LCs were significantly reduced in aged mice in comparison to young control (2-month-old mice) (Fig. 1a). This was further verified by immunofluorescence staining against langerin in gingival cross sections (Fig. 1b). Time-course analysis revealed that a significant reduction in LC frequencies can be already seen in 12-month-old mice (Fig. S1B). A decrease in the frequencies and numbers of LCS in aged mice was also evident in the buccal epithelium (Fig. S2A, B), and in the superficial cervical LNs that drain the oral mucosa (Fig. S2D). Of note, as recently reported, ${ }^{33}$ the level of $\gamma \delta T$ cells, the other major population in the epithelium, declined greatly in aged gingiva (Fig. 1a). Despite the reduction of total gingival $\mathrm{LCs}$ in aged mice, the relative frequencies of LC subsets: LC1: $\mathrm{CD}_{103^{+}} \mathrm{LCs}$ and LC2: $C D 103^{\text {neg }} \mathrm{CX}_{3} \mathrm{CR} 1^{\text {neg }} \mathrm{LCs}$ (both derived from pre-DCs), and moLC: $\mathrm{CX}_{3} \mathrm{CR} 1^{+} \mathrm{LCs}$ (derived from monocytes) were not altered by age (Fig. 1C). To directly compare oral and epidermal LCs during aging, we analyzed in the same mice epidermal LCs using flow cytometry and immunofluorescence analyses. In agreement with previous reports, $^{23,24}$ a considerable reduction of epidermal LCs (MHCII ${ }^{+}$ langerin ${ }^{+}$) was found in aged mice compared with young controls (Fig. 1d, e). Of note, unlike gingival LCs, all the $\mathrm{MHClI}^{+}$cells in young and aged epidermis express langerin, and no $\mathrm{MHCll}^{+}$ langerin ${ }^{-}$cells were detected (Fig. 1d)

Next, we investigated whether mucosal LCs undergo morphological alterations with age alike epidermal LCs. The quantification of morphological parameters was achieved by automated image analysis based on software originally designed for analyzing neurons and neurites (HCA-vision). Acquired whole mount immunofluorescence images were transformed to digital images and parameters such as cell body size, dendrites, and branches were quantified. As depicted in Fig. 2a, b, epidermal LCs undergo major morphological changes in aged mice. This includes a larger 
a
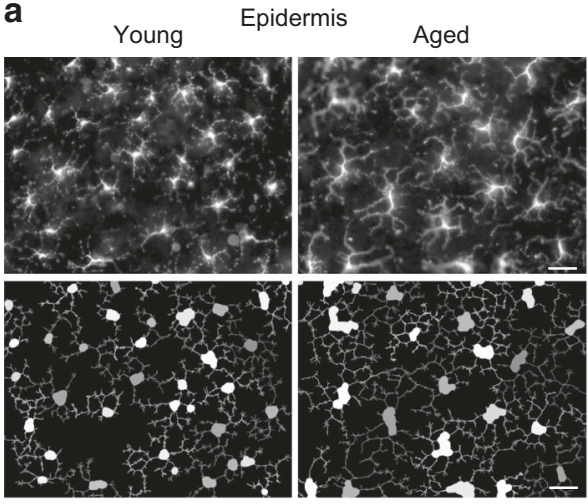

C

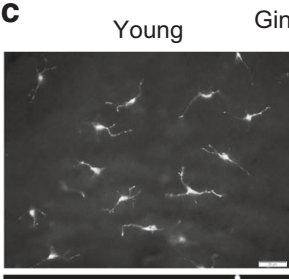

\section{ingiva}

Aged
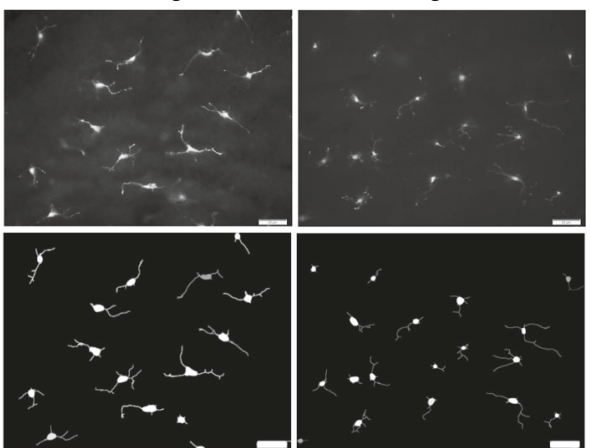

b

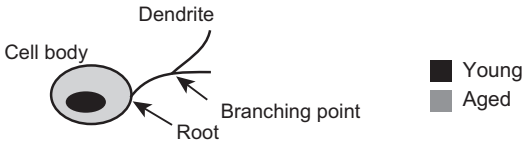

Total dendrite length
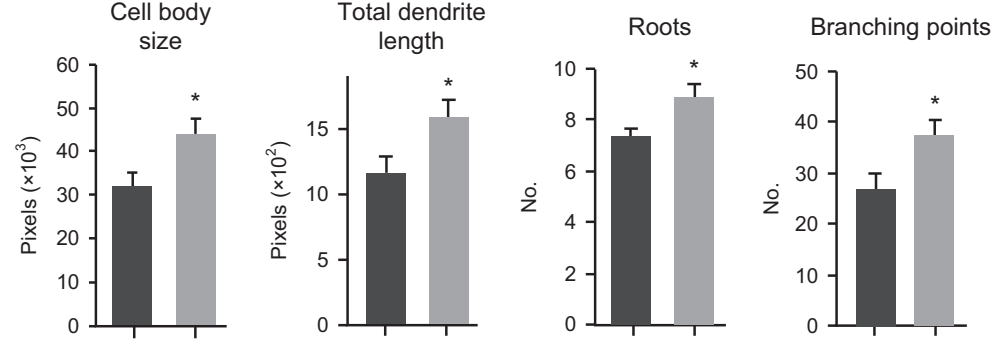

d

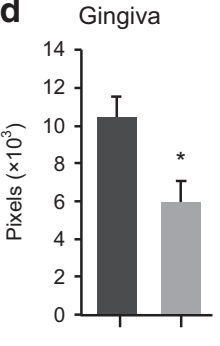

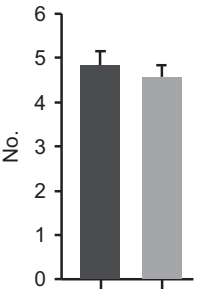

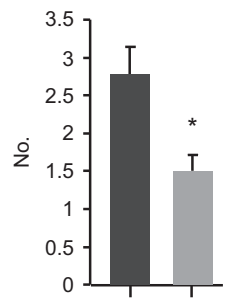

Fig. 2 Dissimilar morphology to aged gingival and epidermal LCs. a Epidermal and c gingival epithelial sheets were collected from young and aged mice and stained with mAbs directed against langerin (green) and MHCll (red). Representative images of immunofluorescence staining (upper panel) and their digital conversion (lower panel). Graphs show the size of the cell body, total dendrite length, numbers of dendrite roots, and branching points in the epidermis (b) and gingiva (d) using the automated image analysis software HCA-Vision. Data are representative of three independent experiments with 3 mice/group. Scale bar $50 \mu \mathrm{m}$. ${ }^{*} P<0.05$ (unpaired Student's $t$ test) compared with young samples.

cell body, higher total dendrite length as well as increased numbers of dendrite roots and branches. Gingival LCs also undergo morphological alterations in aged host, however, the changes were completely opposite from those observed in the epidermis. Aged gingival LCs had smaller cell body size; they exhibited shorter total dendrite length while their dendrites were less branched than young LCs (Fig. 2c, d). Taken together, although the frequencies of both mucosal and epidermal LCs are decreased with age, the LCs undergo contrasting morphological changes according to their respective tissues.

Dysregulated BMP7 and TGF- $\beta 1$ expression in aged epithelia To better understand how aging affects gingival LCs, we examined the expression of two factors that are critically involved in the development of LCs. We first analyzed TGF- $\beta 1$ which is expressed in the epithelium and regulates the differentiation and activation/ maturation state of LCs. 2,10,11,14,34 Using immunofluorescence staining of gingival cross sections we found a slight reduction in the levels of TGF- $\beta 1$ in gingiva of 18-month-old mice in comparison to 2-month-old mice (young control) (Fig. 3a). A western blot analysis further demonstrated a significant reduction in the mature but not precursor form of TGF- $\beta 1$ (Fig. 3b). Quantification of tgfb1 mRNA levels also demonstrated that expression of TGF- $\beta 1$ in the gingiva epithelium is significantly decreased with age (Fig. 3d). Next, we examined the expression of BMP7 that is restricted to the lamina propria and was shown to enables translocation of LC precursors to the epithelium. ${ }^{2}$ As demonstrated in Fig. 3a, b, BMP7 was significantly elevated in aged mice at both protein and mRNA levels in comparison to young mice (Fig. 3a, b). We then analyzed expression of both cytokines in the aged epidermis, and found that expression of TGF- $\beta 1$ was not altered in aged epidermis (Fig. 3c, d). Yet, regardless of the age of the mice, whereas most gingival TGF- $\beta 1$ was present in its mature dimer form (about $25 \mathrm{kDa}$ ), in the epidermis the majority of the protein appeared to be in a precursor form (about $45 \mathrm{kDa}$ ) (Fig. 3b, c). As for BMP7, similar to the gingiva the expression of this protein was significantly upregulated in aged epidermis but only at the protein level (Fig. 3c, d). Collectively, these results suggest that BMP7 and TGF$\beta 1$ are differentially regulated in the gingiva with age, a process that could alter the development of LCs. In the epidermis, on the other hand, only BMP7 is upregulated during aging.

\section{Elevated gingival inflammation and dysregulated microbiota in aged mice}

As sentinels of the epithelium, LCs are responsive to changes in the immunological status of the tissue, thus we compared immunological parameters in the gingiva of 18- and 2-monthold mice. Despite the decrease of gingival LCs and $\gamma \delta T$ cells in aged mice (Fig. 1a), ${ }^{33}$ the frequencies of total CD $45^{+}$leukocytes in aged gingiva were significantly higher than those detected in young mice (Fig. 4a). Neutrophils were predominantly elevated in the aged gingiva, whereas the levels of monocytes were reduced (Figs. 4a and S3). Next, quantification of cytokine expression by RTPCR demonstrated that whereas pro-inflammatory cytokines (Tnfa and $/ 117 a)$ and chemokines $(\mathrm{Cl} / 2$ and $\mathrm{CCl} 20$ ) were upregulated in aged gingiva, expression of anti-inflammatory genes such as $/ / 10$ and Foxp3 was downregulated compared with young mice (Fig. 4b). Furthermore, the mean fluorescence intensity of MHCll on the surface of antigen presenting cells $\left(\mathrm{CD} 11 \mathrm{c}^{+} \mathrm{MHClI}^{+}\right.$cells) 

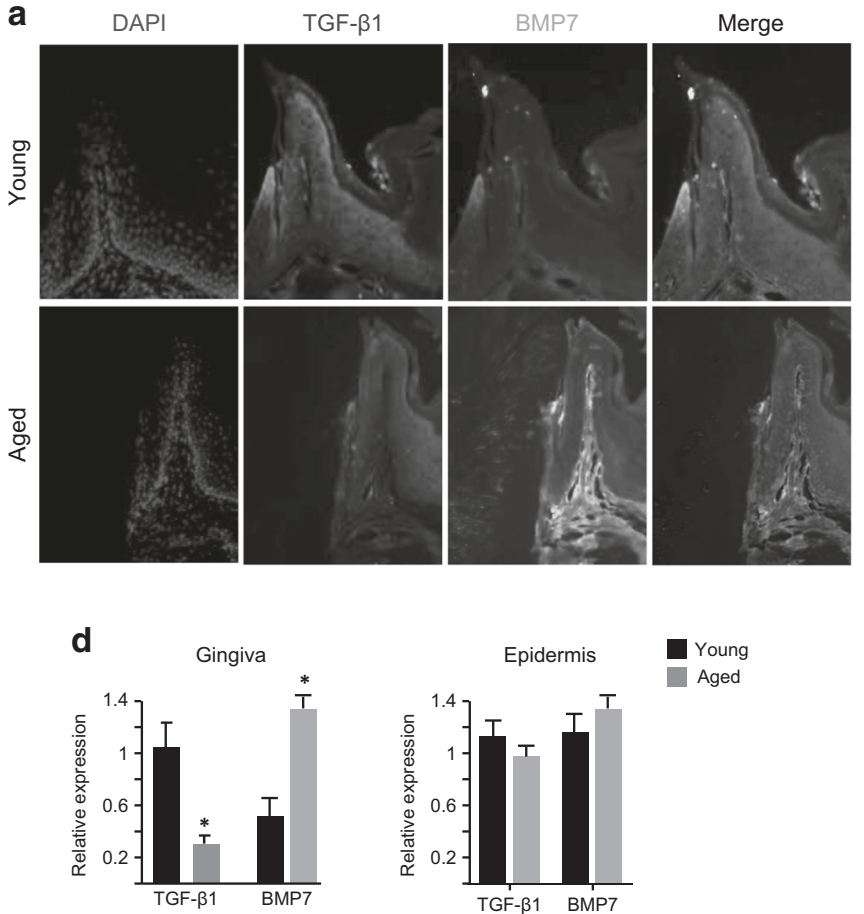

b
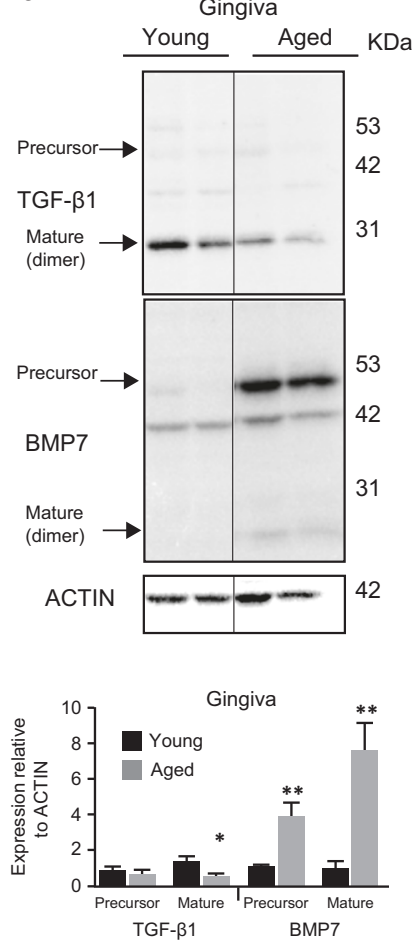

C
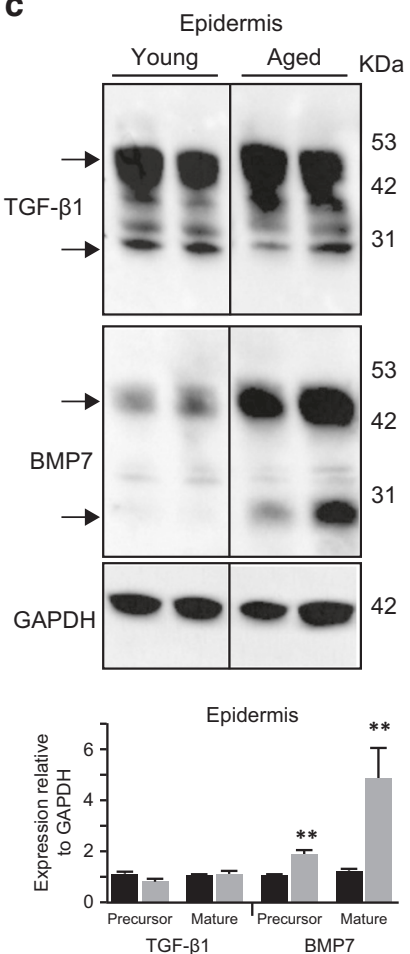

Fig. 3 Expression of TGF- $\beta 1$ and BMP7 is differentially regulated in aged gingiva. a Immunofluorescence staining of maxilla cross sections of young and aged B6 mice with mAbs directed against TGF- $\beta 1$ (red), BMP7 (green), and with DAPI (blue). Representative of three independent experiments. Scale bar $50 \mu \mathrm{m}$. b Western blot analysis showing the presence of TGF- $\beta 1$ and BMP7 in lysates of total gingival epithelial or lamina propria cells, respectively, of young and aged mice. Arrows depict the location of the precursor vs mature dimer form of each protein. ACTIN was used as a control protein. Graph shows the expression intensity of the precursor and mature dimmer forms of TGF- $\beta 1$ and BMP7, the values were normalized to ACTIN and are the mean \pm SEM. Data were collected from four independent experiments with 2-4 mice/group. c Western blot analysis demonstrates the presence of TGF- $\beta 1$ and BMP7 in epidermis lysates of young and aged mice, GAPDH was used as a control protein. Graph shows the expression intensity of the precursor and mature dimmer forms of TGF- $\beta 1$ and BMP7, the values were normalized to GAPDH and are the mean \pm SEM. Data were collected from two independent experiments with 4 mice/group. $\mathbf{d}$ Relative expression of $T g f b 1$ and $B m p 7$ genes in young and aged gingiva or epidermis was quantified by RT-PCR. Graphs present the transcript levels normalized to control group depicted as the mean values + SEM $(n=5)$. Representative data of four independent experiments. ${ }^{*} P<0.05$, ${ }^{* *} P<0.01$ (unpaired Student's $t$ test) compared with young samples.

was significantly upregulated in aged gingiva, suggesting a higher maturation state (Fig. 4c). To examine if such elevated maturation will also result in an accelerated migration to the $\mathrm{LN}$, we painted the oral mucosa of young and aged mice with FITC solution and quantified $\mathrm{FITC}^{+}$migratory DCs/LCs $\left(\mathrm{MHCII}^{\mathrm{hi}} \mathrm{CD} 11 \mathrm{C}^{\mathrm{int}}\right.$ ) in the $\mathrm{LN}$. Indeed, as demonstrated in Fig. 4d, higher percentages of $\mathrm{FIC}^{+}$ DCs/LCs were found in aged mice. In a different experiment we further examined the apoptosis levels of gingival LCs in aged and young mice and revealed in both groups negligible apoptosis levels (Fig. 4e). In contrast to the gingiva, we could not detect any significant alteration in the expression levels of pro- and antiinflammatory genes in the epidermis of young and aged mice (Fig. 4f). Moreover, the epithelium of young and aged mice contains only LCs and $\gamma \delta$ T cells with no evidence of other inflammatory leukocytes (Fig. 4g). Yet, surface expression level of MHCII was slightly but significantly higher in aged than in young LCs (Fig. 4h).

Next, we examined whether the immunological shift found in the gingiva of aged mice is associated with dysregulation of oral microbiota. We first sampled bacteria using oral swabs from the oral cavity of individual sex-matched 2- and 18-month-old B6 mice that were housed under similar conditions. DNA was then extracted and the ratio of ribosomal $16 \mathrm{~S} / 18 \mathrm{~S}$ genes was quantified to compare the microbial load in these mice. As demonstrated in Fig. $5 \mathrm{a}$, the oral cavity of aged mice and young controls sheltered similar bacterial load. We further compared the load of oral anaerobic and aerobic bacteria in these mice, by plating oral swab samples on blood agar under anaerobic or aerobic conditions, and found comparable bacterial numbers among the two groups of mice (Fig. 5b). To test if bacterial diversity was nevertheless changed in aged mice, we performed a taxonomic analysis of the oral microbiota. Alpha diversity analysis indicated that aged and young mice have similar taxa richness in the oral microbiota (Fig. 5c). Moreover, the taxa present in these two groups of mice did not vary significantly as indicated by an unweighted beta diversity and distance analyses (Fig. 5d). Nevertheless, detailed examination revealed that whereas no significant difference was found in phylum diversity (Fig. $5 \mathrm{e}$ ), the relative abundance of certain bacteria was altered in aged mice (Fig. 5f, g). Specifically, the genera Aggregatibacter (Gamaproteobacteria) and Curvibacter (Betaproteobacteria) were expanded in aged mice (Fig. $5 \mathrm{~g}$ ). The absolute abundance, as determined by RT-PCR, of Prevotella (Bacteroidetes) and Lactobacillus (Firmicutes) was elevated in aged mice, whereas Streptococcus (Firmicutes) was not altered (Fig. 5h). We further sampled young and aged mice from a different animal facility and found that although the oral microbiota comprised different bacteria, no significant changes were observed in the bacterial load (Fig. S4). With regard to bacterial diversity, the Proteobacteria phylum was increased in aged mice, and on the family level Streptococcaceae was decreased in aged mice, whereas Pasteurellaceae expanded (Fig. S4B-C). Taken together, these data suggest that aging is accompanied by an elevated gingival inflammation that has moderate impact on the microbial diversity but is associated with expansion of pro-inflammatory 
a
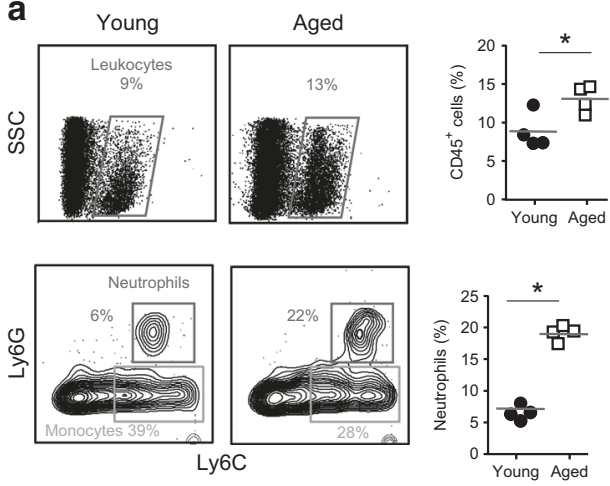

f

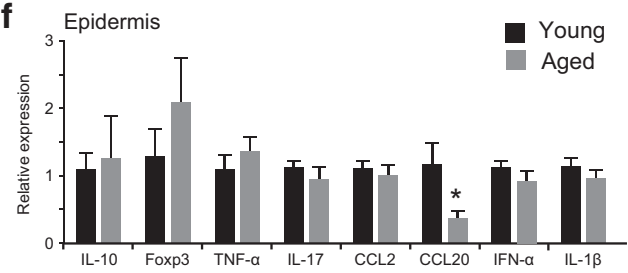

b

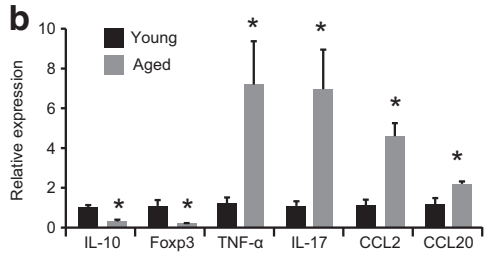

C

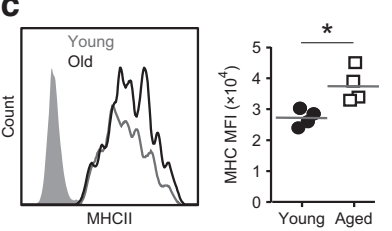

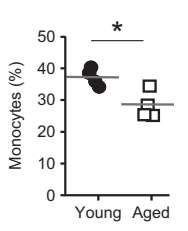

d

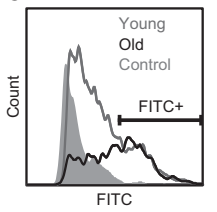

g

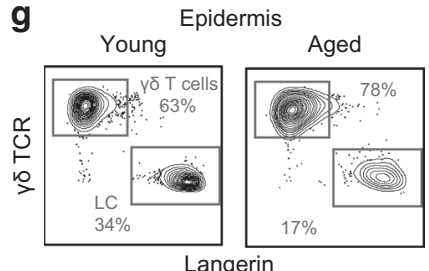

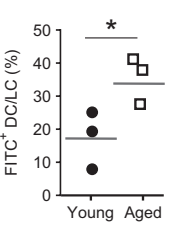

e
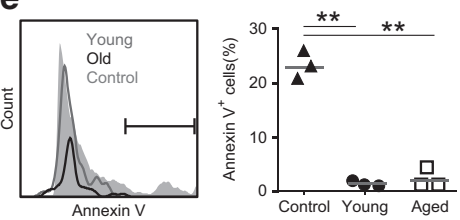

h Epidermis
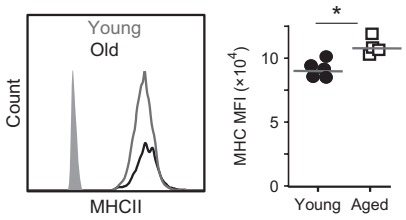

Fig. 4 Elevated inflammatory milieu in aged gingiva. a Representative FACS plots and graphs show the frequencies of total CD45 ${ }^{+}$ leukocytes, neutrophils, and monocytes (pre-gated on $C D 45^{+} \mathrm{CD} 11 \mathrm{~b}^{+}$) in aged and young gingiva. Data are representative of three independent experiments with 4 mice/group + SEM. b Relative mRNA levels of the noted genes in the gingiva of young and aged mice using quantitative RT-PCR. Graph presents the transcript levels normalized to young group depicted as the mean values + SEM $(n=5)$. c Representative FACS histogram present MHCIl expression on gingival APCs of young and aged mice and graph shows the mean fluorescence intensity (MFI). Representative data of two independent experiments with 5 mice/group + SEM. d The oral mucosa of young and aged mice were painted with FITC solution for 2 consecutive days, representative FACS plots and graph show the frequencies of FITC ${ }^{+}$migratory DCs/LCs in the superficial cervical LN $24 \mathrm{~h}$ later. Migratory DC/LCs of the deep cervical LN were used as a negative control. Representative data of two independent experiments with 3 mice/group + SEM. e Apoptosis levels of LCs of young and aged mice, FACS plots and graph show the frequencies of annexin V-positive LCs or gingival epithelial cells (positive control) and present the mean of 3 mice/group + SEM. $f$ Graph presents the relative mRNA transcript levels of the noted genes in the epidermis of young and aged mice normalized to young group depicted as the mean values + SEM $(n=5)$. g Representative FACS plots depict the frequencies of langerin-positive (LCs) and $\gamma \delta T$ cells among total CD45 $5^{+}$leukocytes in young and aged epidermis. $\mathbf{h}$ Representative FACS histogram present MHCII expression on gingival LCs of young and aged mice, and graph shows the mean fluorescence intensity (MFI). Representative data of two independent experiments with 5 mice/ group + SEM. Data are representative of three independent experiments with 5 mice/group + SEM. ${ }^{*} P<0.05$ (unpaired Student's $t$ test) compared with young samples.

bacteria. In addition, gingival DCs and LCs of aged gingiva display higher maturation state and migrate more rapidly to the LN compared with young control.

The microbiota regulates the frequencies of gingival LCs in aged mice

We have shown recently that the microbiota is required for optimal postnatal differentiation of oral LCs, since lower numbers of LCs were found in germ-free (GF) mice few weeks after birth compared with SPF mice. ${ }^{2}$ To examine whether the microbiota also regulates LCs during aging, we analyzed 14-month-old GF mice (aged GF). As demonstrated in Fig. 6a, the epithelium of aged GF mice contains higher frequencies of LCs in comparison to age-matched SPF mice. In fact, the aged GF mice had even higher percentages of $\mathrm{LCs}$ and $\mathrm{APCs}\left(\mathrm{MHClI}{ }^{+} \mathrm{CD} 11 \mathrm{c}^{+}\right)$than 2-months old SPF. The total numbers and frequencies of gingival LCs in aged GF mice were also higher than young GF and SPF mice (Fig. 6a, b), with aged GF displaying a typical LC morphology as in young SPF mice (Fig. 6c). Two-way ANOVA analysis revealed that both microbiota and age significantly regulated gingival LCs. We further revealed that the microbiota significantly modulates inflammatory parameters of the gingiva, as neutrophils and expression of proinflammatory cytokines and chemokines were reduced in aged GF mice compared with SPF mice (Fig. S5A, B). Interestingly, mRNA levels of TGF- $\beta$ and BMP7 were also relatively lower in the gingival epithelium of GF mice despite the high number of LCs in the tissue (Fig. S5B). To examine whether the microbiota has a direct or indirect impact on gingival LCs during aging, we treated aged
SPF mice with broad-spectrum antibiotics for 5 weeks. Such a period of time should be sufficient to reveal changes of LC frequencies, as gingival LCs are capable to completely reestablish their numbers within 3 weeks after depletion or irradiation. ${ }^{3}$ As depicted in Fig. S6, due to the antibiotic treatment the oral microbial load was significantly reduced as well as the percentages of neutrophils and the mRNA levels of TNF- $a$ and CXCL2. Nevertheless, the frequencies of LCs were not changed in the aged gingiva (Fig. 6d), suggesting that the influence of the microbiota on LCs is likely to be indirect. We next analyzed the epidermal LCs in GF mice. In contrast to the gingiva, epidermal LCs were still reduced in 14-month-old GF mice epidermis compared with young SPF and GF mice, though their frequencies were slightly higher than the age-matched SPF control (Figs. 6e and S5C). Moreover, statistical analysis indicated that the age but not microbiota affects LCs in aged epidermis. Since epidermal LCs of aged GF mice seem to be relatively small in the immunofluorescence images (Fig. S5C), we use flow cytometry to test this point. As depicted in Fig. S5D, epidermal LCs of aged GF and young SPF mice had similar distribution based on size and granularity, thus ruling out this possibility. Further examination also revealed that MHCIl expression on aged epidermal LCs was elevated in GF mice as in SPF mice (Fig. S5E), and statistical analysis further verified that age but not microbiota regulates MHCII levels on epidermal LCs. Collectively, these data suggest that LCs are accumulating in the gingiva of GF mice with age, whereas the microbiota plays a role in the reduction of gingival but not epidermal LCs during aging. This effect of the microbiota appears to be indirect and 

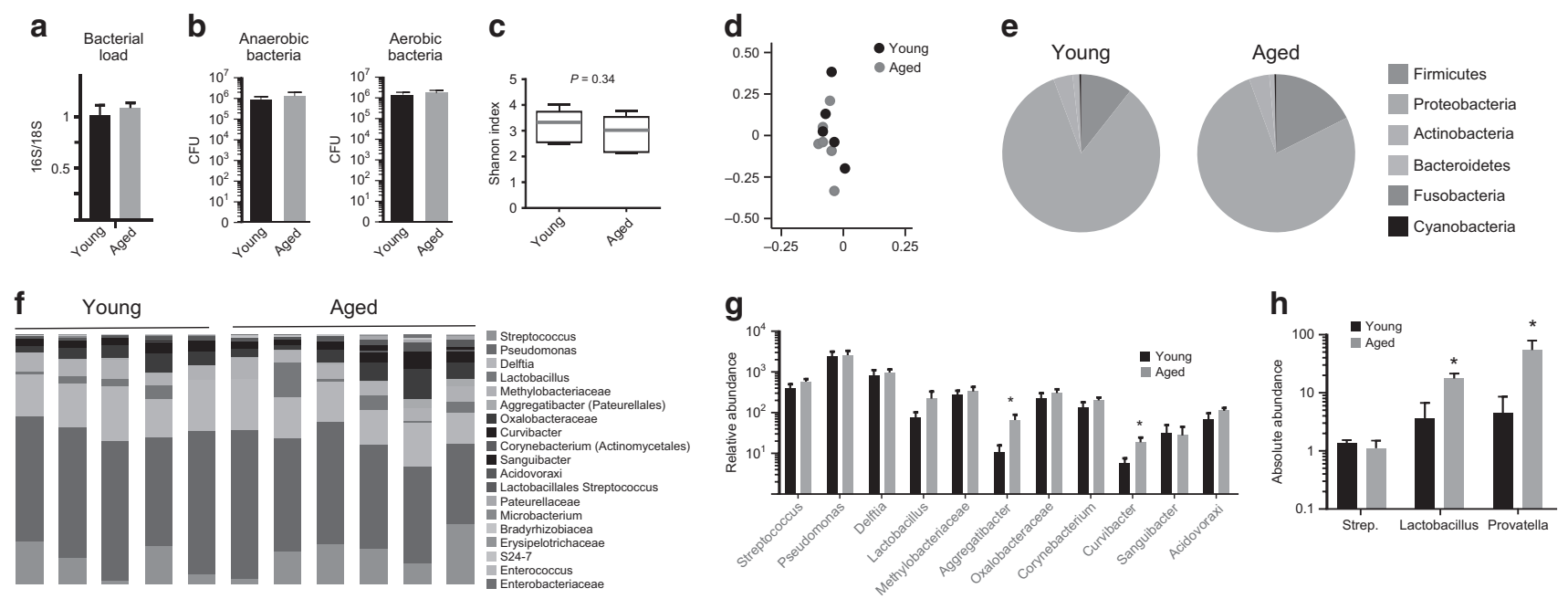

Fig. 5 Aging is marginally affecting the diversity of oral microbiota. a Total oral bacterial load was determined in oral swabs taken from young and aged mice by quantitative RT-PCR of the $16 \mathrm{~S}$ rRNA gene. Bar graphs present the $16 \mathrm{~S} / 18 \mathrm{~S}$ ratio in each group as the mean values + SEM ( $n=8$ per group). b CFU data of total cultivable oral anaerobic and aerobic bacteria sampled from young and aged mice. Representative data of one of two independent experiments with 8 mice/group + SEM. c-f Relative abundance of taxa in oral swabs sampled from young and aged mice. c $\alpha$-diversity plot representing taxa richness in samples of both groups of mice (rarified to 6200 reads). d Principal coordinates analysis of weighted UniFrac distances based on $16 \mathrm{~S}$ rRNA of both groups. e Pie charts representing the mean distribution of bacterial phyla among young and aged mice. $\mathbf{f}$ Histograms for individual mice represent the distribution of sequences in operational taxonomic units (OTUs) assigned to each family. g Relative abundance of high abundance bacterial families. Taxonomic data from one of two independent experiments are shown with 5-6 mice per group + SEM. h Absolute abundance of certain bacterial families in oral swabs collected from young and aged mice using quantitative RT-PCR analysis. Representative data of one of three independent experiments with 5 mice/group + SEM. ${ }^{*} P<0.05$ (unpaired Student's $t$ test) compared with young samples.

could be mediated via the epithelium, which controls LC development while being itself regulated by the microbiota.

BM precursors of aged mice present an intact capacity to differentiate to mucosal LCs

We next examined if the reduction of gingival LCs in aged mice could also result due to an intrinsic impairment of aged BM precursors to differentiate into LCs. First, quantification of pre-DCs $\left(\mathrm{CD} 45^{+} \mathrm{Lin}^{\text {neg }} \mathrm{CD} 11 \mathrm{C}^{+} \mathrm{MHCII}{ }^{\text {neg }} \mathrm{FLT3}^{+}\right.$SIRP-a ${ }^{\text {int }}$ ), the predominant precursors of gingival LCs, revealed that pre-DCs are present at higher percentages in 18-month-old BM compared with 2-monthold BM (Fig. 7a). Macrophage/DC precursors (MDP) $\left(C D 45^{+} \operatorname{Lin}{ }^{\text {neg }} C^{-}\right.$

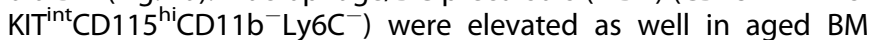
(Fig. 7b). Similarly, we found higher percentages of classical monocytes $\left(\mathrm{CD} 45^{+} \mathrm{Lin}^{\text {neg }} \mathrm{C}-\mathrm{KIT}^{\mathrm{neg}} \mathrm{CD} 115^{\text {hi }} \mathrm{CD} 11 \mathrm{~b}^{+} \mathrm{Ly} 6 \mathrm{C}^{+}\right)$, the second progenitor of gingival LCs, and common monocytes precursors (cMoP) $\left(\mathrm{CD} 45^{+} \mathrm{Lin}^{\text {neg }} \mathrm{C}-\mathrm{KIT}{ }^{\text {int }} \mathrm{CD} 115^{\mathrm{hi}} \mathrm{CD} 11 \mathrm{~b}^{-} \mathrm{Ly}_{6 \mathrm{C}} \mathrm{C}^{+}\right)$ with age (Fig. $7 \mathrm{~b})$. This suggests that despite the reduction of gingival LCs with age, mucosal LC precursors are more frequent in aged mice. To assess if these aged precursors are capable to differentiate into LCs, aged and young BM cells were cultured for 5 days with GM-CSF and TGF- $\beta 1$. Using flow cytometry, we found that aged $\mathrm{BM}$ cells were able to acquire a LC-like phenotype $\left(\mathrm{CD}_{4} 5^{+} \mathrm{MHCII}^{+} \mathrm{CD} 11 \mathrm{c}^{+} \mathrm{EpCAM}^{+} \mathrm{CD}_{205^{+}}\right.$) (Fig. 7c). In fact, aged BM cells had a superior capacity to differentiate to LC-like cells in comparison to young BM, probably due to the higher precursor frequencies they contain. We next examined the capacity of aged and young BM cells to differentiate to gingival LCs in vivo using mixed BM chimeras. BM cells were collected from 18-month-old CD45.2 $2^{+}$mice and 2-month-old $\mathrm{CD}_{4} 5.1^{+}$mice and identical numbers of BM cells $\left(1 \times 10^{6}\right.$ cells) from young and aged mice (1:1 ratio) were adoptively transferred into lethally irradiated CD $45.2^{+}$ or CD45.1 $1^{+}$18-month-old and 2-month-old hosts, respectively (Fig. 7d). To monitor leukocyte reconstitution in the chimeric mice, we analyzed by flow cytometry the frequencies of circulating $B$ cells and monocytes, as representatives of the lymphoid and myeloid compartments, respectively. In both aged and young hosts, the fraction of $B$ cells derived from young donors outnumbered $B$ cells purified from aged donors (Fig. 7e), in line with the reported reduced differentiation capacity of lymphoid cells with age. ${ }^{35}$ Monocytes showed a different picture since in aged host the monocytes from aged donors were higher than young monocytes, concurring with the elevated frequencies of their precursors $\mathrm{CMOP}$ in aged BM. In young host, the young monocytes recovered better compared with aged host, but remained still significantly lower than aged monocytes. We then checked the contribution of these aged and young BM precursors to the LC population in the gingiva. Flow cytometry analysis revealed that in young hosts the BM precursors from both donors differentiated similarly to LCs (Fig. 7f), indicating that aged BM precursors have intact ability to differentiate to gingival LCs. Interestingly, in aged host, gingival LCs originated mainly from aged precursors, a result mirroring the precursor frequencies in the circulation, suggesting that aged myeloid cells are better adapted to aged host than young myeloid cells. Collectively, these findings strongly suggest that aged LC precursors have an intact intrinsic capability to differentiate into LCs. Moreover, the myeloid biased found in aged host further support LC development from aged precursors.

\section{DISCUSSION}

This study demonstrates that even though the frequencies of LCs in the epidermis and gingiva are reduced during aging, the mechanisms mediating this phenomenon in both epithelia are distinct. We provided evidence that in aged mice the differentiation potential of mucosal LC precursors is intact or even superior to that observed in young mice. Nevertheless, the higher inflammation observed in aged gingiva leads to rapid maturation of LCs and subsequently their accelerated migration to the lymph nodes. $^{36}$ Indeed, the morphology of aged gingival LCs as well as their elevated $\mathrm{MHCll}$ expression are typical characteristics of mature LCs. ${ }^{37}$ This accelerated maturation and migration in aged mice resemble the dynamics of oral LCs in young mice upon acute 
a Gingiva
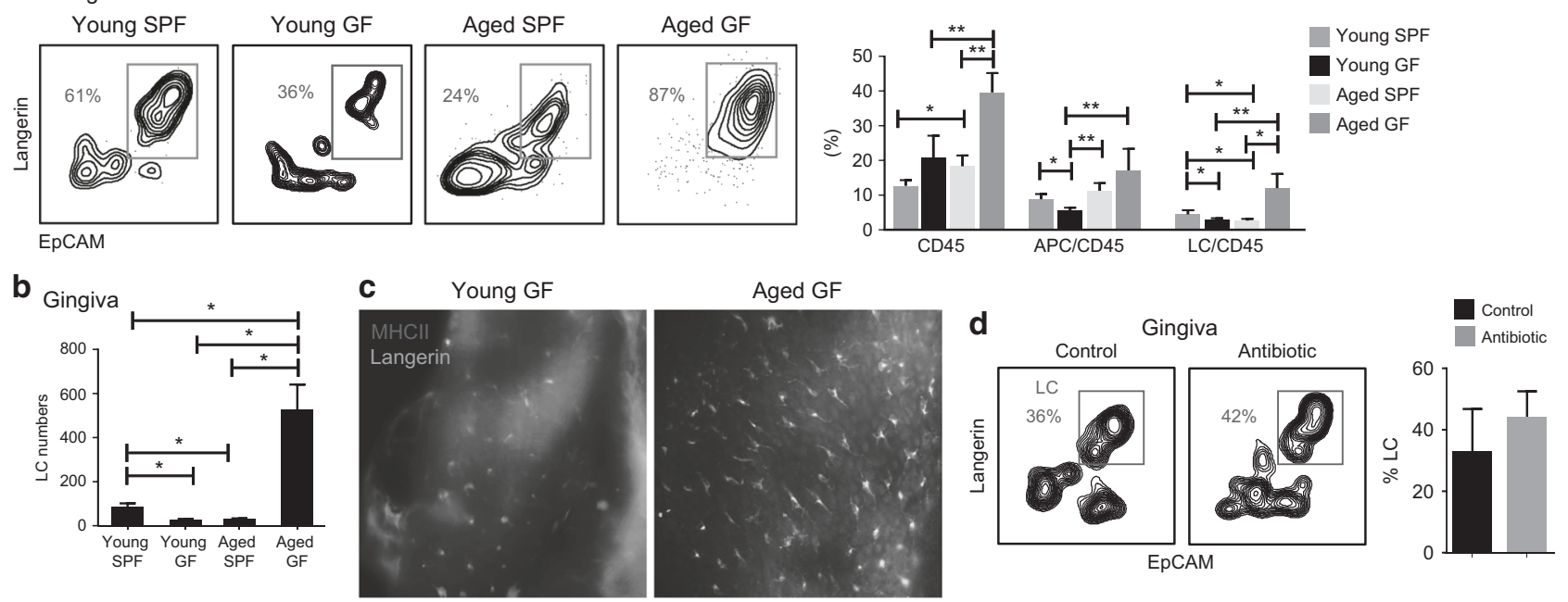

e Epidermis
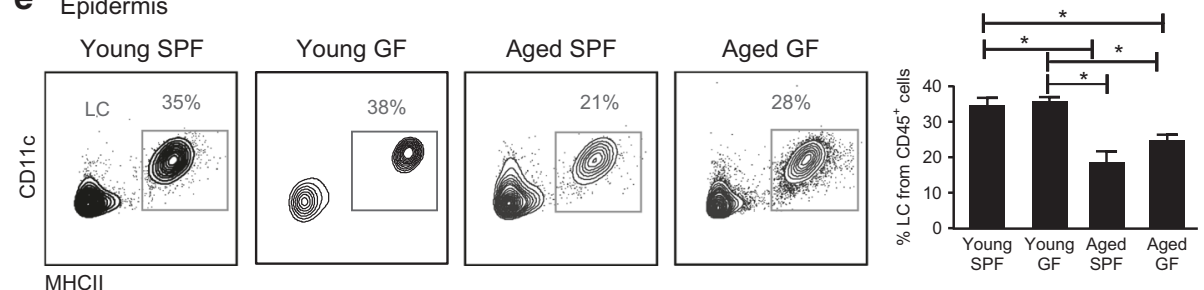

Fig. 6 Aged gingival LCs are shaped by the microbiota. a Representative FACS plots show the frequencies of gingival LCs in young SPF, young GF, aged SPF, and aged GF mice. Graph presents the percentages of CD45 leukocytes, APC $\left(\mathrm{MHCII}{ }^{+} \mathrm{CD} 11 \mathrm{c}^{+}\right)$and LCs in the gingival epithelium. Representative data of two independent experiments with 4-5 mice/group + SEM. $\mathbf{b}$ Total numbers of LCs in the gingiva of aged and young GF and SPF mice using flow cytometry analysis. Data are representative of two independent experiments with 5 mice/group + SEM. c Immunofluorescence staining of gingival epithelial sheets of young and aged GF mice with mAbs directed against langerin (green) and MHCII (red). d Aged SPF mice were treated with broad-spectrum antibiotics in the drinking water for 5 weeks. Representative FACS plots and graph show the frequencies of LCs in antibiotic-treated mice and the control group. Data of one out of two independent experiments with 5 mice/group + SEM. e Representative FCAS plots and graphs present the frequencies of epidermal LCs in young and aged SPF and GF mice. Data of one out of two independent experiments with 5 mice/group + SEM. ${ }^{*} P<0.05$, ${ }^{*} P<0.01$ (two-way ANOVA with Bonferroni correction).

oral inflammation. ${ }^{38}$ In the skin, on the contrary, the epidermis does not become more inflamed during aging, and epidermal LCS are also not showing an activated morphology/phenotype, but rather expand their size and dendrites to monitor larger epidermal areas as a compensatory mechanism to their reduced numbers. It worth mentioning, that in humans aging is associated with increased inflammation in the epidermis. ${ }^{39}$ Of note, previous studies reported that young and aged epidermal LCs have comparable apoptosis and proliferation kinetics, characteristics that define their local maintenance by self-renewal. ${ }^{23,24}$ This suggests that unlike gingival LCs, the reduced levels of LCs in the epidermis might result from a deficiency in local LC progenitors.

The equal contribution of pre-DCs and monocytes to the various LC subsets in aged and young gingiva is in line with the increased frequencies of both precursors with age. On this regard, biased differentiation of hematopoietic stem cells (HSCs) toward myeloid cells in aged mice ${ }^{40,41}$ including circulating monocytes ${ }^{42}$ has been shown previously. A recent study further reported that lymphoid progenitors are not declining with age but rather undergo changes in gene expression with a myeloid-biased genetic profile, ${ }^{35}$ The microbiota was also proposed as a factor in the age-associated myeloid bias. ${ }^{43,44}$ Here, we directly demonstrate using up-to-date phenotypic markers that CMoP, MDP, and pre-DCs are more frequent in the BM compartment during aging. Interestingly, mixed BM chimeric mice indicated that aged myeloid precursors (represented by monocytes) are more adapted to aged host than young precursors, confirming the emerging view that cell-extrinsic in addition to cell-intrinsic factors, regulate the myeloid bias of HSCs with age. ${ }^{45}$ Aged B cells, on the other hand, failed to reach the levels of young $B$ cells in the circulation of either young or aged host, suggesting that autonomous factors play a central role in B-cell differentiation during aging. This, however, should be taken with caution since our analysis was performed 3 weeks after transplantation and a previous study reported that only 14 weeks post transplantation aged B cells reach similar levels to young $B$ cells; yet, the age of the host was not clearly depicted in the study. ${ }^{46}$ With regard to LC differentiation, it has been reported that aging is associated with low TGF- $\beta 1$ levels, which increase systemic inflammation (Inflamm-aging) and facilitate myeloid over lymphoid differentiation of HSCs. ${ }^{47}$ In reference to this scheme, it is likely that the reduction of TGF- $\beta 1$ we observed in aged gingiva facilitates the differentiation of LCS from myeloid precursors on one hand, whereas on the other hand it elevates local inflammation and accelerates LC maturation. This might also explain the reduced frequencies of monocytes in the aged epithelium, which could be explained by their rapid differentiation into LCs in attempt to restore the reduced LC population. Unlike TGF- $\beta 1$, BMP7 levels were significantly increased in the lamina propria of aged mice. This could represents a compensatory mechanism to the reduced numbers of LCs, since BMP7 is required for translocation of LC precursors to the epithelium, ${ }^{2}$ and upregulation of BMP7 facilitates recruitment of new precursors. It will be interesting to examine in the future if TGF- $\beta 1$ and BMP7-related intracellular signaling such as activation of Smad proteins is altered in the aged gingival epithelium as was reported to aged epidermis. ${ }^{48}$ 
a

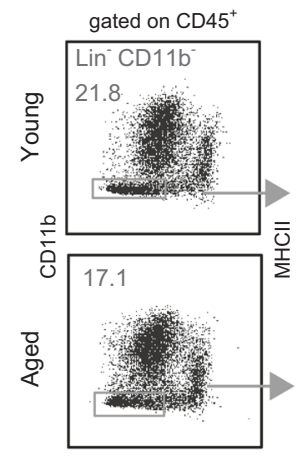

Lineage

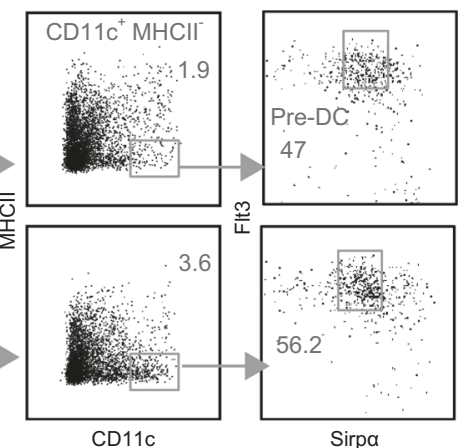

CD11c

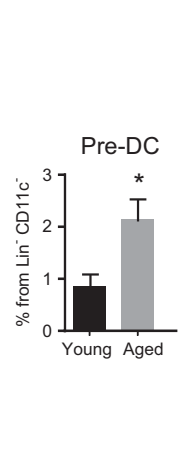

C

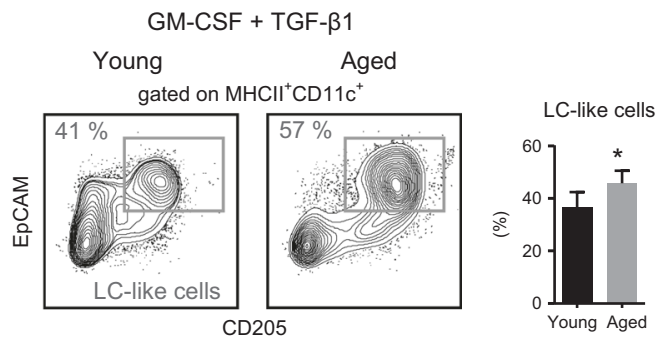

b
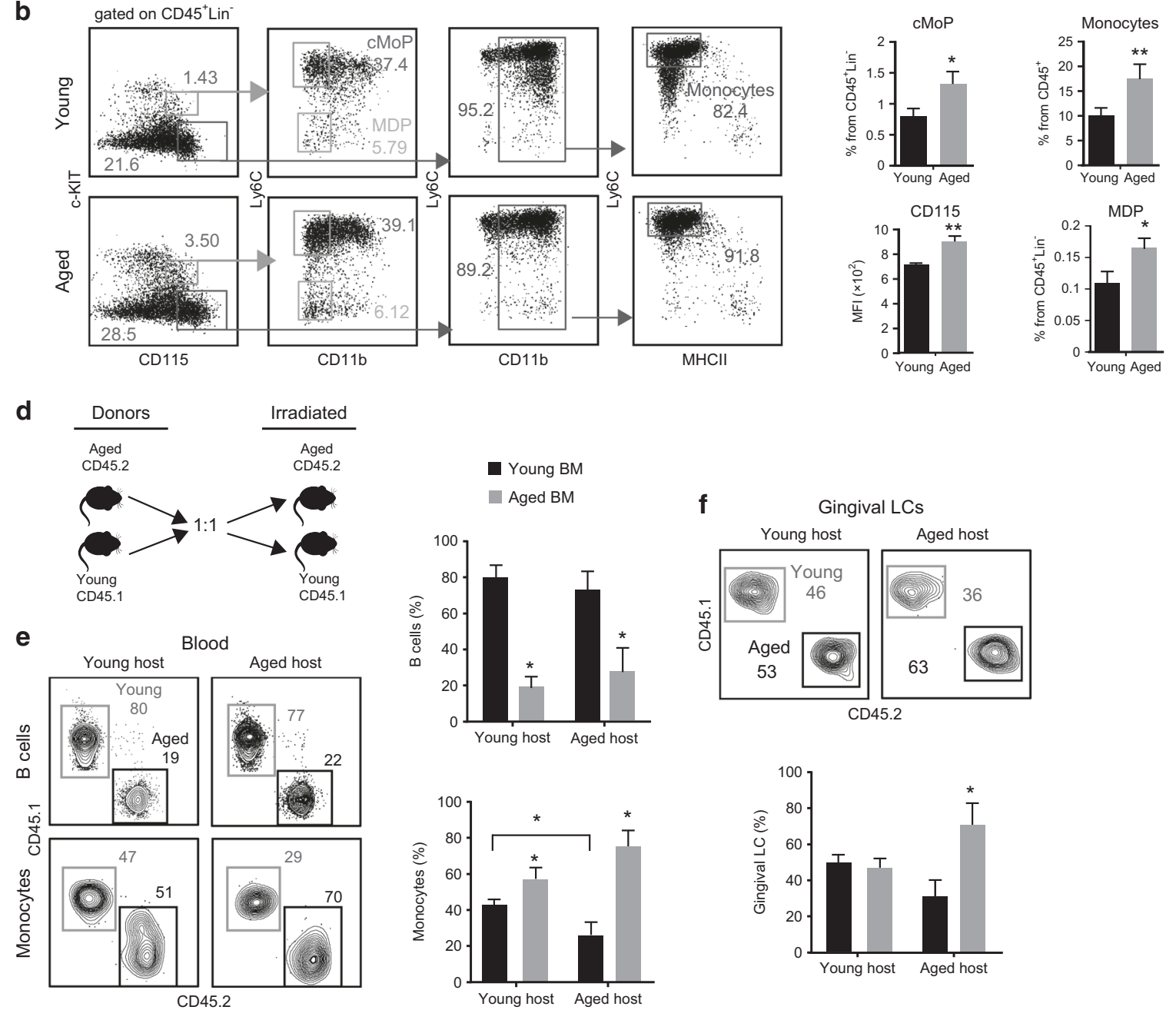

Fig. 7 Aged BM precursors efficiently differentiated to gingival LCs. Representative FACS plots and graph show the gating strategies and frequencies of (a) pre-DCs and (b) common monocytes precursors (cMoP), monocytes and macrophage/DC precursors (MDP) in young and aged BM. Data are representative of three independent experiments with 5 mice/group + SEM. c Young and aged BM cells were cultured with GM-CSF and TGF- $\beta 1$, FCAS plots and graph show the percentages of LC-like cells $\left(\mathrm{MHCII}^{+} \mathrm{CD} 11 \mathrm{C}^{+} \mathrm{EpCAM}{ }^{+} \mathrm{DEC} 205^{+}\right)$in the cultures. Representative data of three independent experiments with 3-5 mice/group. d CD45.2 $2^{+}$aged mice and CD45.1 ${ }^{+}$young mice were lethally irradiated and then transplanted with a mixture $(1: 1)$ of BM cells from CD45.2 $2^{+}$aged and CD45.1 $1^{+}$young mice, the mice were analyzed 3 weeks after transplantation. e Representative FACS plots and graphs show the percentages of CD45. $1^{+}$vs. CD45.2 $2^{+}$B lymphocytes and monocytes in the blood of aged and young host. $f$ FACS plots and graph demonstrate the frequencies of gingival LCs originating from young CD45.1 $1^{+}$vs. aged CD45.2 $\mathrm{BM}$ precursors in the gingival epithelium of young and aged host. Data present the mean values of 5 mice/group + SEM. ${ }^{*} P<0.05,{ }^{* *} P<0.01$ (unpaired Student's $t$ test) compared with young samples.

We have shown previously that initial development of gingival LCs is regulated by the microbiota, since few weeks after birth GF mice had less LCs compared with SPF mice. ${ }^{2}$ In the present study, however, aged GF mice had higher levels of LCs compared with aged SPF, and actually even more than young SPF and GF mice, indicating that eventually GF mice reach optimal LC levels. Due to the absence of bacteria, LCs of GF mice are likely to be less activated and thus they accumulate in the sterile gingiva, 
explaining their higher frequencies compared with young SPF. The absence of the microbiota can also explain the reduced TGF- $\beta 1$ and BMP7 levels in the gingiva of aged GF vs. aged SPF mice, similar to the situation we previously reported in young SPF and GF mice, ${ }^{2}$ thus representing the steady-state expression pattern of GF mice. In contrast to the gingiva, epidermal LCs were reduced in aged GF mice, indicating minimal impact of the microbiota on their ontogeny throughout life. This corroborates our previous work showing that the microbiota is also not essential for postnatal differentiation of epidermal LCs. ${ }^{2}$

Our study suggests that aging has a minimal impact on the load and diversity of the oral microbiota. These results are similar to the situation in humans, as no major differences were detected in the oral microbiota of young and elderly individuals. ${ }^{42,49,50}$ Nevertheless, certain groups of bacteria were expanded in aged mice, most likely due to their capability to utilize inflammatory by-product for anaerobic respiration as we and others have shown. ${ }^{51-53}$ With regards to Streptococcal bacteria which are usually reduced under inflammatory conditions, we found either no change or a reduction in their abundance that appear to be related to the overall bacterial composition. Similarly, in humans, Streptococcal bacteria are left unchanged in elderly people and only Actinomyces strains that can expand in inflammatory milieu were found in elevated levels. ${ }^{49,50}$ This suggests that age-related gingival inflammation is not sufficient to induce major alterations in the oral bacterial diversity, in contrast to periodontal inflammation induced by a pathogen or a ligature which substantially alters the oral microbiota. ${ }^{54,55}$

In summary, we demonstrate that although mucosal LC precursors are more frequent during aging, aged mice have reduced gingival LCs most likely due to the indirect effect of the microbiota on the mucosal epithelium with age. This supports the emerging view on the central role of the niche on the differentiation of mucosal LCs. ${ }^{4}$ Since LCs are critical regulators of oral mucosal immunity, their reduction with age might have major clinical implications. LCs play an important role in regulating host immunity to the dental biofilm, and dysregulated activation of these cells during aging might accelerate periodontitis and the associated systemic conditions. ${ }^{56}$ Moreover, LCs are considered to be the first APCs to encounter early stages of oral squamous cell carcinoma and to mount anti-tumor immunity. Their reduced numbers with age could explain the higher risk of elderly people to develope this type of oral cancer, which represent about $90 \%$ of all oral malignancies. ${ }^{57}$ Further investigations are required to elucidate the clinical impact of age-associated reduction of oral LCs in order to provide healthy aging.

\section{MATERIAL AND METHODS}

Mice

CD45.2 ${ }^{+}$C57BL/6 (B6) mice were purchased from Harlan (Rehovot, Israel). $\mathrm{CD} 45.1^{+} \mathrm{B} 6$ mice were purchased from the Jackson Laboratory (Bar Harbor, ME). The mice were maintained under SPF condition. Young mice were analyzed between 2 and 3 months of age and aged mice between 18 and 24 months of age, unless described else in the text. All animal protocols were approved by the Hebrew University Institutional Animal Care and Ethics Committee. Aged GF C57BL/6 mice at the age of 14 months were maintained in sterile isolators at the Weizmann Institutes, the GF experiments were approved by the Institutional Animal Care and Usage Committee of the Weizmann Institute of Science.

\section{Chimeras}

BM chimeras were generated by transplanting an equal mixture of B6 CD45.1 $1^{+}$and B6 CD45.2 $2^{+}$BM cells purified from young and aged $\mathrm{C} 57 \mathrm{BL} / 6$ mice, respectively, into lethally irradiated (950 rad) aged and young mice. The mice were left to rest for 3 weeks to enable the reconstitution of LCs and then the chimerism was examined in the gingiva and blood samples as indicated in the text.

In vitro LC differentiation cultures

The femur was isolated from the mice, cleaned from soft tissues in sterile RPMI 1640 and soaked in $70 \%$ ethanol for $1 \mathrm{~min}$ for sterilization. The femur was then washed with sterile PBS $\times 1$ and the bone ends were removed by sterile scissors. BM cells were eluted from the bone by flushing them several times using a sterile syringe filled with RPMI 1640, and the cells were then washed, treated with ACK solution for $3 \mathrm{~min}$ on ice, washed again and counted. BM cells $\left(5 \times 10^{5}\right.$ cells/well) in 24-well plates (Nunc) were cultured with complete RPMI medium [450 ml RPMI 1640, 50 $\mathrm{ml}$ fetal calf serum (FCS), $5 \mathrm{ml}$ L-glutamine, $50 \mu \mathrm{M} \beta$-mercaptoethanol, penicillin $(100 \mathrm{U} / \mathrm{ml})$, streptomycin $(100 \mu \mathrm{g} / \mathrm{ml})$ and gentamicin $(50 \mu \mathrm{g} / \mathrm{ml})]$ supplemented with GM-SCF $(20 \mathrm{ng} / \mathrm{ml})$ and TGF- $\beta 1(10 \mathrm{ng} / \mathrm{ml})$ for 5 days to induce their differentiation. The cells were washed and stained with the noted antibodies for flow cytometry analysis.

\section{Statistical analysis}

Data were expressed as means \pm SEM. Statistical tests were performed using the one-way analysis of variance (ANOVA) and Student's $t$ test, a $P$ value of $<0.05$ was considered significant. For studies involving young and aged GF and SPF mice (two independent variances), significant differences were analyzed using two-way ANOVA with Bonferroni correction and were depicted as ${ }^{*} P<0.05$ and ${ }^{* *} P<0.01$.

\section{ACKNOWLEDGEMENTS}

The work was supported by grant from the Israel Science Foundation 766/16 to A.H.H and by a grant from the Israeli Ministry of Science 3-13993.

\section{AUTHOR CONTRIBUTIONS}

A.H.H. and A.W. conceived of the study. E.E. and H.B. help designed the studies and supplied materials. Y.H., R.S. initiated the study and M.N., T.C., Y.S., O.B., K.Z., D.M., L.E.B. and A.L. performed the experiments and analyze the data while H.S., S.H., G.B.C. and $\mathrm{O} . \mathrm{H}$. helped with implementation. All authors contributed to refinement of the study protocol and approved the final manuscript.

\section{ADDITIONAL INFORMATION}

The online version of this article (https://doi.org/10.1038/s41385-020-0301-y) contains supplementary material, which is available to authorized users.

Competing interests: The authors declare no competing interests.

Publisher's note Springer Nature remains neutral with regard to jurisdictional claims in published maps and institutional affiliations.

\section{REFERENCES}

1. Romani, N., Clausen, B. E. \& Stoitzner, P. Langerhans cells and more: langerinexpressing dendritic cell subsets in the skin. Immunol. Rev. 234, 120-141 (2010).

2. Capucha, T. et al. Sequential BMP7/TGF-beta1 signaling and microbiota instruct mucosal Langerhans cell differentiation. J. Exp. Med. 215, 481-500 (2018).

3. Capucha, T. et al. Distinct murine mucosal langerhans cell subsets develop from pre-dendritic cells and monocytes. Immunity 43, 369-381 (2015).

4. Hovav, A. H. Mucosal and skin Langerhans cells - nurture calls. Trends Immunol. 39, 788-800 (2018).

5. Hoeffel, G. et al. Adult Langerhans cells derive predominantly from embryonic fetal liver monocytes with a minor contribution of yolk sac-derived macrophages. J. Exp. Med. 209, 1167-1181 (2012).

6. Chorro, L. et al. Langerhans cell (LC) proliferation mediates neonatal development, homeostasis, and inflammation-associated expansion of the epidermal LC network. J. Exp. Med. 206, 3089-3100 (2009).

7. Ginhoux, F. et al. Langerhans cells arise from monocytes in vivo. Nat. Immunol. 7, 265-273 (2006) 
8. Sere, K. et al. Two distinct types of Langerhans cells populate the skin during steady state and inflammation. Immunity 37, 905-916 (2012).

9. Ferrer I. R., et al. A wave of monocytes is recruited to replenish the longterm Langerhans cell network after immune injury. Sci Immunol. 4, 1-10 (2019).

10. Borkowski, T. A., Letterio, J. J., Farr, A. G. \& Udey, M. C. A role for endogenous transforming growth factor beta 1 in Langerhans cell biology: the skin of transforming growth factor beta 1 null mice is devoid of epidermal Langerhans cells. J. Exp. Med. 184, 2417-2422 (1996).

11. Kaplan, D. H. et al. Autocrine/paracrine TGFbeta1 is required for the development of epidermal Langerhans cells. J. Exp. Med. 204, 2545-2552 (2007).

12. Tenno, M. et al. Cbfbeta2 deficiency preserves Langerhans cell precursors by lack of selective TGFbeta receptor signaling. J. Exp. Med. 214, 2933-2946 (2017).

13. Yasmin, N. et al. Identification of bone morphogenetic protein 7 (BMP7) as an instructive factor for human epidermal Langerhans cell differentiation. J. Exp. Med. 210, 2597-2610 (2013).

14. Zahner, S. P. et al. Conditional deletion of TGF-betaR1 using Langerin-Cre mice results in Langerhans cell deficiency and reduced contact hypersensitivity. J. Immunol. 187, 5069-5076 (2011).

15. Garcovich, S. et al. Skin cancer epidemics in the elderly as an emerging issue in geriatric oncology. Aging Dis. 8, 643-661 (2017)

16. Laube, S. Skin infections and ageing. Ageing Res. Rev. 3, 69-89 (2004).

17. Wanke, I. et al. Staphylococcus aureus skin colonization is promoted by barrier disruption and leads to local inflammation. Exp. Dermatol. 22, 153-155 (2013).

18. Davidson, B. J., Root, W. A. \& Trock, B. J. Age and survival from squamous cell carcinoma of the oral tongue. Head Neck 23, 273-279 (2001).

19. Lasisi, T. J., Oluwasola, A. O., Lasisi, O. A. \& Akang, E. E. Association between langerhans cells population and histological grade of oral squamous cell carcinoma. J. Oral. Maxillofac. Pathol. 17, 329-333 (2013).

20. Takahara, M. et al. Stromal CD10 expression, as well as increased dermal macrophages and decreased Langerhans cells, are associated with malignant transformation of keratinocytes. J. Cutan. Pathol. 36, 668-674 (2009).

21. Upadhyay, J., Rao, N. N. \& Upadhyay, R. B. A comparative analysis of langerhans cell in oral epithelial dysplasia and oral squamous cell carcinoma using antibody CD-1a. J. Cancer Res Ther. 8, 591-597 (2012).

22. Kindt, N. et al. Langerhans cell number is a strong and independent prognostic factor for head and neck squamous cell carcinomas. Oral. Oncol. 62, 1-10 (2016)

23. $\mathrm{Xu}, \mathrm{Y}$. P. et al. Aging affects epidermal Langerhans cell development and function and alters their miRNA gene expression profile. Aging 4, 742-754 (2012).

24. Sprecher, E. et al. Effect of aging on epidermal dendritic cell populations in C57BL/6J mice. J. Investig. Dermatol. 94, 247-253 (1990).

25. Pilkington, S. M. et al. Lower levels of interleukin-1 beta gene expression are associated with impaired Langerhans' cell migration in aged human skin. Immunology 153, 60-70 (2018).

26. Bhushan, M. et al. Tumour necrosis factor-alpha-induced migration of human Langerhans cells: the influence of ageing. Br. J. Dermatol. 146, 32-40 (2002).

27. Cruchley, A. T. et al. Langerhans cell density in normal human oral mucosa and skin: relationship to age, smoking and alcohol consumption. J. Oral. Pathol. Med. 23, 55-59 (1994).

28. Sasaki, M. Histomorphometric analysis of age-related changes in epithelial thickness and Langerhans cell density of the human tongue. Tohoku J. Exp. Med. 173, 321-336 (1994).

29. Zavala, W. D. \& Cavicchia, J. C. Deterioration of the Langerhans cell network of the human gingival epithelium with aging. Arch. Oral. Biol. 51, 1150-1155 (2006).

30. Bodineau, A. et al. Do Langerhans cells behave similarly in elderly and younger patients with chronic periodontitis? Arch. Oral. Biol. 52, 189-194 (2007).

31. Rittman, B. R., Hill, M. W., Rittman, G. A. \& Mackenzie, I. C. Age-associated changes in Langerhans cells of murine oral epithelium and epidermis. Arch. Oral. Biol. 32, 885-889 (1987)

32. Raffaniello, R. D. \& Roy, M. Immunohistological analysis of the immune cells in the normal oral mucosa of aging mice. Gerodontology 9, 51-57 (1990).
33. Wilharm, A., et al. Mutual interplay between $\mathrm{IL}-17$ producing $\gamma \delta \mathrm{T}$ cells and microbiota orchestrates oral mucosal homeostasis. Proc Natl Acad Sci USA. 116, 2652-2661 (2019).

34. Bobr, A. et al. Autocrine/paracrine TGF-beta1 inhibits Langerhans cell migration. Proc. Natl Acad. Sci. USA 109, 10492-10497 (2012).

35. Montecino-Rodriguez, E. et al. Lymphoid-biased hematopoietic stem cells are maintained with age and efficiently generate lymphoid progeny. Stem Cell Rep. 12, 584-596 (2019).

36. Kissenpfennig, A. et al. Dynamics and function of Langerhans cells in vivo: dermal dendritic cells colonize lymph node areas distinct from slower migrating Langerhans cells. Immunity 22, 643-654 (2005).

37. Seite, $\mathrm{S}$. et al. Alterations in human epidermal Langerhans cells by ultraviolet radiation: quantitative and morphological study. Br. J. Dermatol. 148, 291-299 (2003).

38. Arizon, M. et al. Langerhans cells down-regulate inflammation-driven alveolar bone loss. Proc. Natl Acad. Sci. USA 109, 7043-7048 (2012).

39. Kinn, P. M. et al. Age-dependent variation in cytokines, chemokines, and biologic analytes rinsed from the surface of healthy human skin. Sci. Rep. 5, 10472 (2015).

40. Cho, R. H., Sieburg, H. B. \& Muller-Sieburg, C. E. A new mechanism for the aging of hematopoietic stem cells: aging changes the clonal composition of the stem cell compartment but not individual stem cells. Blood 111, 5553-5561 (2008).

41. Roeder, I. et al. Characterization and quantification of clonal heterogeneity among hematopoietic stem cells: a model-based approach. Blood 112, 4874-4883 (2008)

42. Strohacker, K., Breslin, W. L., Carpenter, K. C. \& McFarlin, B. K. Aged mice have increased inflammatory monocyte concentration and altered expression of cellsurface functional receptors. J. Biosci. 37, 55-62 (2012).

43. Khosravi, A. et al. Gut microbiota promote hematopoiesis to control bacterial infection. Cell Host Microbe 15, 374-381 (2014).

44. Nagai, Y. et al. Toll-like receptors on hematopoietic progenitor cells stimulate innate immune system replenishment. Immunity 24, 801-812 (2006).

45. Elias, H. K., Bryder, D. \& Park, C. Y. Molecular mechanisms underlying lineage bias in aging hematopoiesis. Semin. Hematol. 54, 4-11 (2017).

46. Morrison, S. J., Wandycz, A. M., Akashi, K., Globerson, A. \& Weissman, I. L. The aging of hematopoietic stem cells. Nat. Med. 2, 1011-1016 (1996).

47. Challen, G. A., Boles, N. C., Chambers, S. M. \& Goodell, M. A. Distinct hematopoietic stem cell subtypes are differentially regulated by TGF-beta1. Cell Stem Cell 6, 265-278 (2010)

48. Han, K. H. et al. Alteration of the TGF-beta/SMAD pathway in intrinsically and UVinduced skin aging. Mech. Ageing Dev. 126, 560-567 (2005).

49. Marsh, P. D., Percival, R. S. \& Challacombe, S. J. The influence of denture-wearing and age on the oral microflora. J. Dent. Res. 71, 1374-1381 (1992).

50. Percival, R. S., Challacombe, S. J. \& Marsh, P. D. Age-related microbiological changes in the salivary and plaque microflora of healthy adults. J. Med. Microbiol. 35, 5-11 (1991)

51. Nassar, M. et al. GAS6 is a key homeostatic immunological regulator of hostcommensal interactions in the oral mucosa. Proc. Natl Acad. Sci. USA 114, E337-E346 (2017)

52. Winter, S. E., Lopez, C. A. \& Baumler, A. J. The dynamics of gut-associated microbial communities during inflammation. EMBO Rep. 14, 319-327 (2013).

53. Winter, S. E. et al. Gut inflammation provides a respiratory electron acceptor for Salmonella. Nature 467, 426-429 (2010).

54. Dutzan, N., et al. A dysbiotic microbiome triggers TH17 cells to mediate oral mucosal immunopathology in mice and humans. Sci. Transl. Med. 10, 1-12 (2018).

55. Hajishengallis, G. et al. Low-abundance biofilm species orchestrates inflammatory periodontal disease through the commensal microbiota and complement. Cell Host Microbe 10, 497-506 (2011).

56. Lopez, R., Smith, P. C., Gostemeyer, G. \& Schwendicke, F. Ageing, dental caries and periodontal diseases. J. Clin. Periodontol. 44(Suppl 18), S145-S152 (2017).

57. Vincent-Chong, V. K. et al. Impact of age on disease progression and microenvironment in oral cancer. J. Dent. Res. 97, 1268-1276 (2018). 